EPJ Web of Conferences 71, 00144 (2014)

DOI: 10.1051/epjconf/20147100144

(C) Owned by the authors, published by EDP Sciences, 2014

\title{
Proton femtoscopy in STAR
}

\author{
Hanna Zbroszczyk for the STAR Collaboration ${ }^{1, a}$ \\ ${ }^{1}$ Warsaw University of Technology, Koszykowa 75, 00-662 Warsaw, Poland
}

\begin{abstract}
Two-particle femtoscopy allows one to study the properties of matter created in heavy ion collisions. It makes the study of space-time evolution of the source possible and may be applied to many different combinations of hadron pairs. Two-proton femtoscopy enables to extract the radii of produced sources which, compared to those obtained from pion studies, provide additional information about source characteristics. In this paper we present the correlation functions obtained for protons and antiprotons for $\mathrm{Au}+\mathrm{Au}$ collisions at $\sqrt{s_{N N}}=200 \mathrm{GeV}$.
\end{abstract}

\section{Introduction}

Solenoidal Tracker At RHIC (STAR) is one of experiments performed at Relativistic Heavy Ion Collider (RHIC) at Brookhaven National Laboratory (BNL). The main goal of the STAR experiment is to measure the properties of matter created in heavy ion collisions at ultrarelativistic energies, and to study the formation of matter and the properties of interactions between hadrons. For over ten years, STAR has been collecting data from $\mathrm{Au}+\mathrm{Au}$ collisions at different energies: 7.7, 11.5, 19.6, 27 , 39, 62.4, 130, and $200 \mathrm{GeV}$. The Beam Energy Scan (BES) program [1], which includes colliding energies below $\sqrt{s_{N N}}=62.4 \mathrm{GeV}$, is mainly focused on exploration of the phase transition between hadron gas and quark-gluon plasma, and on the search for the critical point of quantum chromodynamics. The highest of collision energy $\sqrt{s_{N N}}=200 \mathrm{GeV}$ enables measurement of hot and dense matter after sQGP formation.

Analyzing the momentum correlations, it is possible to access information about space-time characteristics of source which cannot be measured directly. Correlations of identical baryons as proton-proton or antiproton-antiproton reflect the properties of the Quantum Statistics (QS) and of the Final State Interactions (FSI): Coulomb and strong. Correlations of proton-antiproton pairs are sensitive to the FSI only. The correlation effect is determined by the distance separating emission points in space and time. In this paper we present results for proton femtoscopy from $\mathrm{Au}+\mathrm{Au}$ collisions at $\sqrt{s_{N N}}=200$ $\mathrm{GeV}$.

Due to high multiplicities of protons, two-proton correlations were intensively measured before at AGS (BNL), SPS (CERN) and SIS (GSI) energies [2]. The STAR measurement, in addition to exploring new collision energies, has the advantage of a simultaneous measurement of all three combinations of protons and antiprotons.

ae-mail: gos@if.pw.edu.pl

This is an Open Access article distributed under the terms of the Creative Commons Attribution License 2.0, which permits unrestricted use, distribution, and reproduction in any medium, provided the original work is properly cited. 


\section{Details of data analysis}

The correlations of two particles are measured in the momentum difference variable $k^{*}=\frac{Q_{\text {inv }}}{2}=$ $\frac{1}{2} \sqrt{\left(p_{1}-p_{2}\right)^{2}-\left(E_{1}-E_{2}\right)^{2}}$. The correlation function is defined as a ratio: $C\left(k^{*}\right)=\frac{A\left(k^{*}\right)}{B\left(k^{*}\right)}$. Pairs of correlated particles which come from the same event enter into the numerator $A\left(k^{*}\right)$ and the pairs of uncorrelated particles from different events into the denominator $B\left(k^{*}\right)$. All particles are registered by the main STAR detector, the Time Projection Chamber (TPC) and the Time of Flight (TOF).

The centrality selection is based on the uncorrected primary charged particle multiplicity in the pseudorapidity region as measured by the TPC detector. The centrality classes are calculated as fraction of this multiplicity distribution. Glauber calculations are performed using a Monte Carlo calculation. The centrality bin defined as $0-5 \%$ corresponds to the most central collisions amounting to $5 \%$ of the total cross section, while $70-80 \%$ is the most peripheral collisions. Almost 200 millions of minimum bias events collected in year 2010 are divided according to the percentage of total cross-section of the collision into three centralities: central (0-10\%), midcentral (10-30\%), and peripheral (30-80\%). All particles are selected using the energy loss in the detector $(d E / d x)$. Protons and antiprotons are chosen in the transverse momentum range: $0.4<p_{T}<2.5 \mathrm{GeV} / \mathrm{c}$ and in the rapidity window $|y|<0.5$. Each track is extrapolated to the primary vertex. If the shortest distance between track and the vertex exceeds $1 \mathrm{~cm}$, the track is discarded. This removes a significant fraction of non-primary track candidates. Information derived from TOF detector allow one to estimate the mass of particle, we require the mass square to be between 0.76 and $1.03 \mathrm{GeV} / \mathrm{c}^{2}$. The particle purity is not taken into account as it is estimated as almost $100 \%$. The effect of tracks splitting and merging is also taken into account.

\section{The estimation of residual correlations}

\subsection{Basics of residual correlation effects}

From the experimental point of view, many secondary protons and antiprotons are indistinguishable from primordial particles, because their parent particles are not detected. Not taking this fact into consideration may lead to misinterpretation of the measured results, where instead of proton-proton interaction the correlations between other particles (which decayed finally into proton) are observed. Two main weak decay channels of interest are considered here:

$$
\begin{gathered}
\Lambda \rightarrow p+\pi^{-} \\
\Sigma^{+} \rightarrow p+\pi^{0}
\end{gathered}
$$

In fact, all residual correlations arising from decay channels which lead to proton (antiproton) through hyperons are considered. In order to evaluate the effect of residual correlations occurring in proton femtoscopy, our analysis uses the Therminator (THERMal Heavy IoN GenerATOR) Monte Carlo event generator [3, 4]. The generator was designed to study particle production at SPS, RHIC and LHC energies. The space-time emission point, as well as parent information is stored for each particle. 


\subsection{Combining contributions from several sources}

The formula on experimentally measured proton - proton (and antiproton-antiproton) correlation is described by the Equation:

$$
\begin{aligned}
C F_{\text {meas }}\left(k^{*}\right)= & C F_{p-p}\left(k^{*}\right) F_{p-p}\left(k^{*}\right)+C F_{p-\Lambda}\left(k^{*}\right) F_{p-\Lambda}\left(k^{*}\right) \\
& +C F_{\Lambda-\Lambda}\left(k^{*}\right) F_{\Lambda-\Lambda}\left(k^{*}\right)+C F_{p-\Sigma}\left(k^{*}\right) F_{p-\Sigma}\left(k^{*}\right) \\
& +C F_{\Sigma-\Sigma}\left(k^{*}\right) F_{\Sigma-\Sigma}\left(k^{*}\right)+C F_{\Lambda-\Sigma}\left(k^{*}\right) F_{\Lambda-\Sigma}\left(k^{*}\right)
\end{aligned}
$$

where $F_{x-y}\left(k^{*}\right)$ represents the fraction of measured proton-proton pairs, where most primary parents were $x$ and $y . C F_{x-y}\left(k^{*}\right)$ means the residual contribution of $\mathrm{x}-\mathrm{y}$ correlation.

The formula for the proton-antiproton system is a little more complicated as it contains all combinations of protons and antiprotons from 6 different groups. We do not describe here the mechanism of calculating the residual correlations existing in proton-antiproton system as it is similar to one for identical proton femtoscopy. The aim of this study is to estimate the pure proton-proton correlation after removing from the measured observables the residual ones. In order to evaluate the fractions $F_{x-y}\left(k^{*}\right)$ of each correlation, all protons are divided into three groups: primordial particles, protons coming from $\Lambda$ hyperons and ones coming from decays of $\Sigma^{+}$baryons. For all two-proton combinations, the $k^{*}$ distributions are computed (Figure 1). The dominant contribution comes from $p-\Lambda$ pairs for all $k^{*}$ values, indicating that $p-\Lambda$ residual interaction strongly affects measured $p-p$ correlation. The presence of other types of residual correlations is much less significant.

The fraction of pure correlation is slightly $k^{*}$ dependent function with the mean value from 0.31 (for the $\bar{p}-\bar{p}$ system) to 0.38 (for the $p-p$ system) due to $\bar{p} / p$ ratio smaller than $\bar{\Lambda} / \Lambda$ ratio. The main week decay channels of protons and anti-protons are lambda and anti-lambda ones. The fraction of pure correlations is shown on Figure 2.

\subsection{Convolution of decay kinematics}

The $p-\Lambda$ correlation function has been measured by STAR [6], as a function of $k_{p-\Lambda}^{*}$. However the argument of $C F_{p-\Lambda}$ in Equation 3 is $k_{p-p}^{*}$, the relative momentum between two protons: in this case, one of the protons is the decay daughter of $\Lambda$. To calculate the $p-\Lambda$ contribution to the measured $p-p$ correlation function, the $p-\Lambda$ correlation function must be convoluted with the $\Lambda$ decay kinematics. Figure 3 shows from which region of $k_{p-\Lambda}^{*}$ the $p-\Lambda$ residual correlation affect which range of $k_{p-p}^{*}$ in experimentally measured $p-p$ correlation. For each value of $k_{p-p}^{*}$, the influence of $p-\Lambda$ correlation is computed as a sum over all $k_{p-\Lambda}^{*}$ bins of experimentally measured $p-\Lambda$ correlation scaled by the factor from $W\left(k_{p-p}^{*}, k_{p-\Lambda}^{*}\right)$ histogram. $p-\Lambda$ correlation cannot be corrected on purity as it contains the residual correlations derived from higher order decays.

$$
C F_{p-\Lambda}\left(k_{p-p}^{*}\right)=\sum_{k_{p-\Lambda}^{*}} C F_{p-\Lambda}^{\text {meas }}\left(k_{p-\Lambda}^{*}\right) W\left(k_{p-p}^{*}, k_{p-\Lambda}^{*}\right)
$$

Residual correlations from $p-\Sigma^{+}$and $\Sigma^{+}-\Sigma^{+}$channels are assumed to arise only from Coulomb interactions for all systems in addition to Quantum Statistics for $\Sigma^{+}-\Sigma^{+}$. The $\left(k_{p-p}^{*}\right.$ and $\left.k_{p-\Sigma^{+}}^{*}\right),\left(k_{p-p}^{*}\right.$ and $\left.k_{\Sigma^{+}-\Sigma^{+}}\right)$kinematic dependencies of decay of $\Sigma^{+}$hyperon are considered separately. 
EPJ Web of Conferences

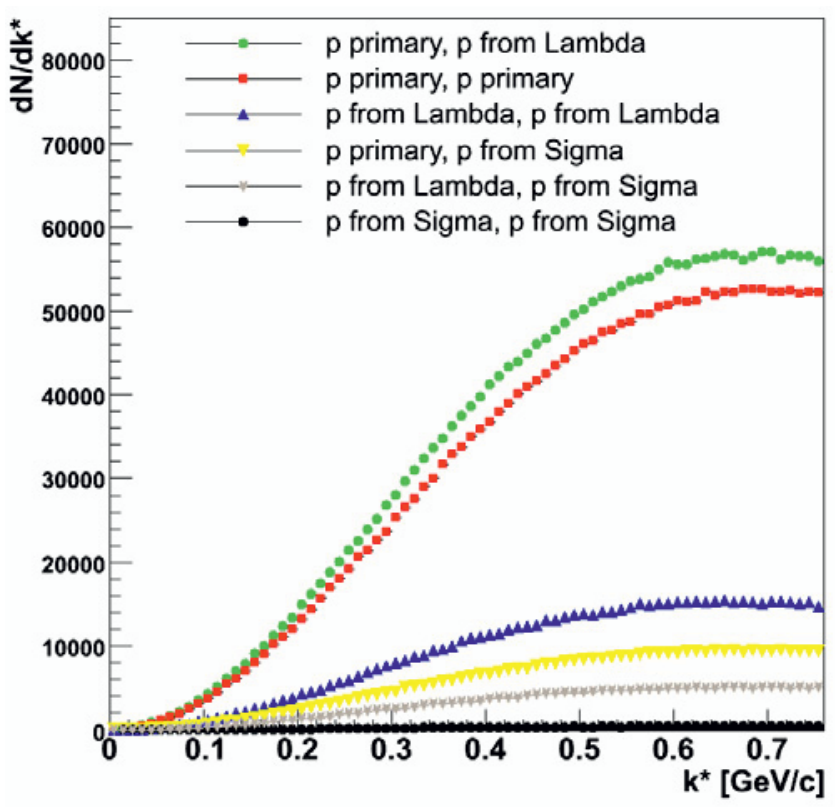

Figure 1. Two-proton distributions of momentum differences in PRF

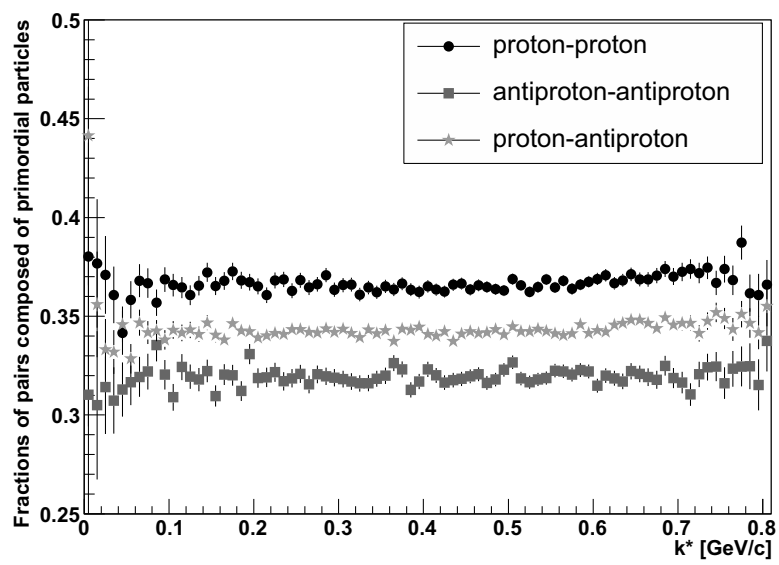

Figure 2. The fraction of pairs composed of primordial particles

\subsection{Effects on extracted length scales}

The net effect of residual correlations corrections is shown in Figure 4 and Figure 5. A first estimate of the fraction of primary-primary proton pairs was 0.25 . (i.e fraction of of primary protons 0.5 ). Finally, the extracted correlation is further reduced when Residual Correlations are also corrected for. The calculations include statistical error bars only. 


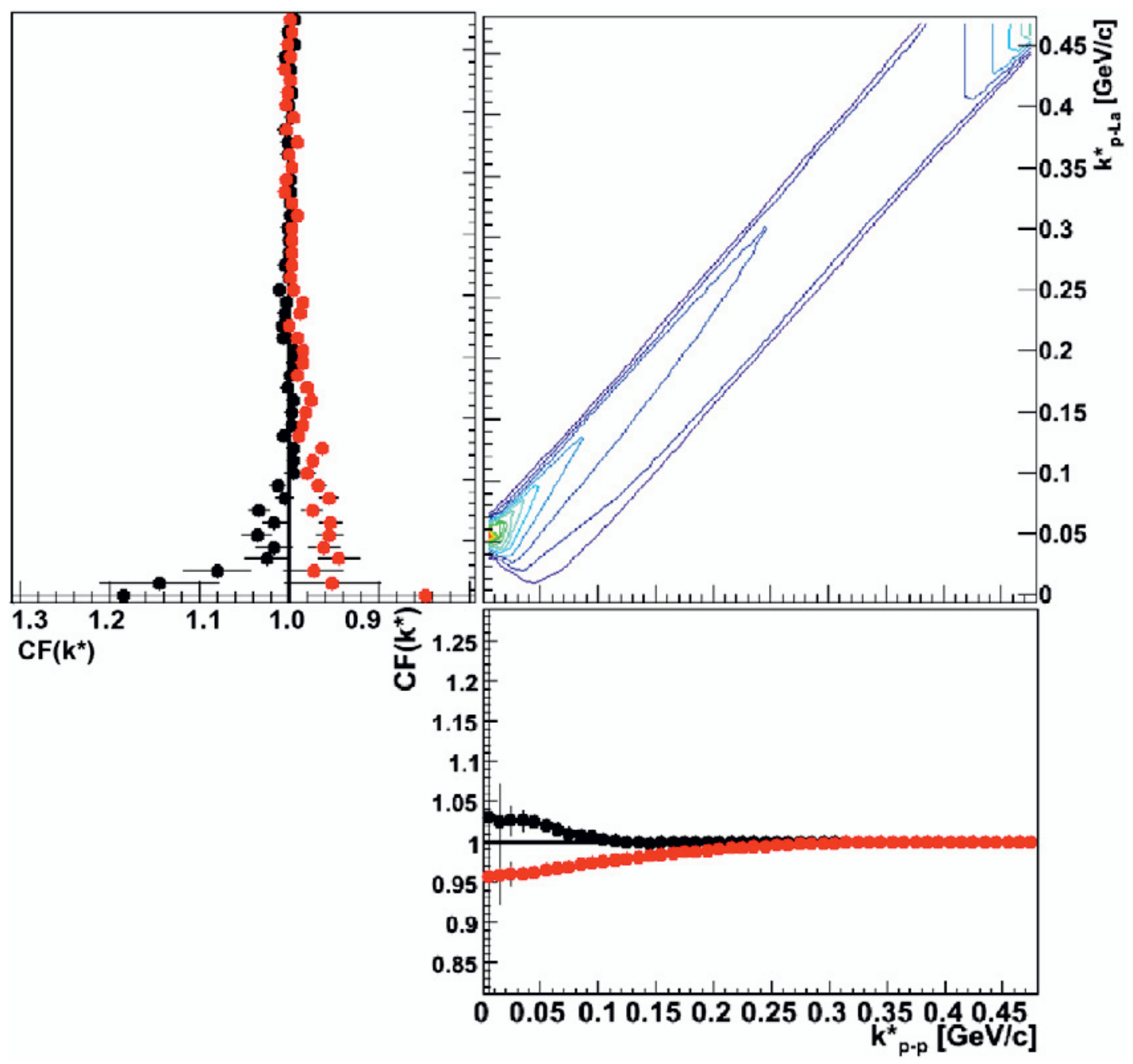

Figure 3. Left-hand side of the top panel illustrates experimentally measured $p-\Lambda$ (black marker) and $\bar{p}-$ $\Lambda$ (red marker) correlation functions for central collisions. Right-hand side of the top panel shows kinematic dependencies $W\left(k_{p-p}^{*}, k_{p-\Lambda}^{*}\right)$ of lambda decays, $\mathrm{k}^{*}$ of $p-\Lambda$ pairs versus $\mathrm{k}^{*}$ of $p-p$ pairs. Right-hand side of the bottom panel exhibits $p-\Lambda$ (black circles) and $\bar{p}-\Lambda$ (red circles) residual correlation functions measured with $p-p, \bar{p}-\bar{p}$ and $p-\bar{p}$ correlations.

Since the $\bar{p}-\Lambda$ correlation is much stronger and wider than $p-\Lambda$ one, the residual effects on the $p-\bar{p}$ system are more significant than those for $p-p$ system. This is clear in Figure 5 , where the effects of the various corrections on $p-\bar{p}$ are shown.

\section{Results for $\mathrm{Au}+\mathrm{Au}$ collisions at $\sqrt{s_{N N}}=200 \mathrm{GeV}$}

Figure 6 presents the measured correlation functions of proton-proton, antiproton-antiproton, and proton-antiproton for three different centralities for collision energy $\sqrt{s_{N N}}=200 \mathrm{GeV}$. Figure 7 shows 


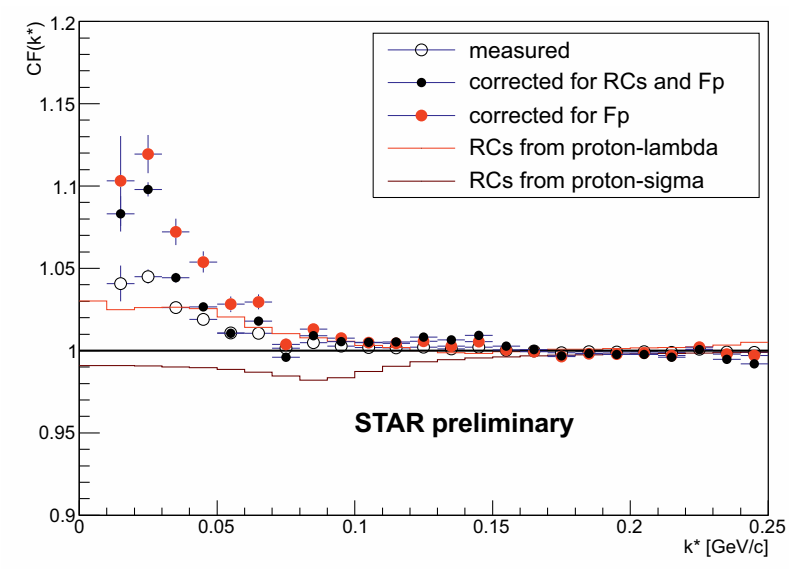

Figure 4. The measured (open symbols), corrected for fraction of primordial particles (red) and corrected for residual fractions correlation functions proton-proton (red), at $\sqrt{s_{N N}}=200 \mathrm{GeV}$ for the most central data. The effect of residual correlations from proton-lambda is indicated by red line and the impact for residual correlations for proton-sigma is marked by dark red line.

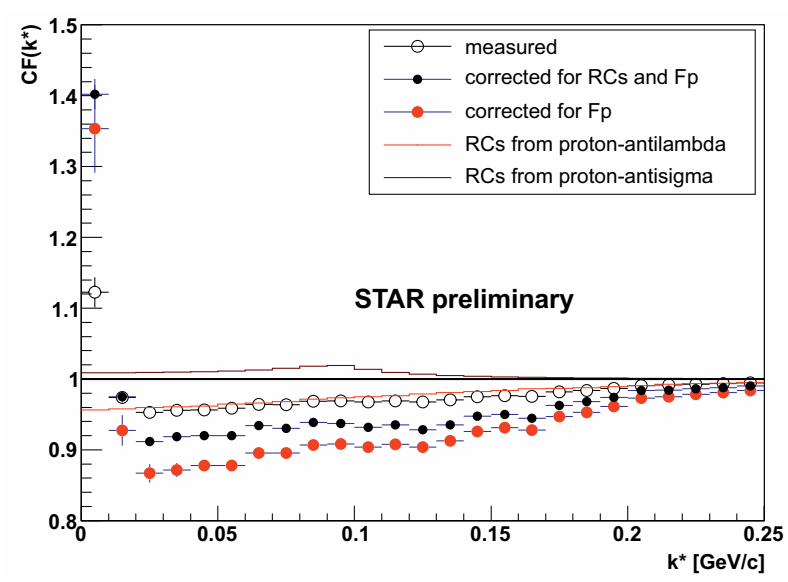

Figure 5. The measured (open symbols), corrected for fraction of primordial particles (red) and corrected for residual correlations correlation functions proton-proton (red), at $\sqrt{s_{N N}}=200 \mathrm{GeV}$ for the most central data. The effect of residual correlations from proton-lambda is indicated by red line and the impact for residual correlations for proton-sigma is marked by dark red line.

the corrected for residual correlations functions for three systems and three centrality of collision. The latter correction accounts for the mutual correlations between hyperons which subsequently decay into protons. It is important because a significant fraction (around 50\%) of (anti)protons originate from such decays. For all systems the width and height of the correlation function decrease - reflecting the source size increase - with centrality. The results indicate that the residual correlations should be applied as it influences strongly the measured correlation functions, as described in details in [5]. 
ICNFP 2013

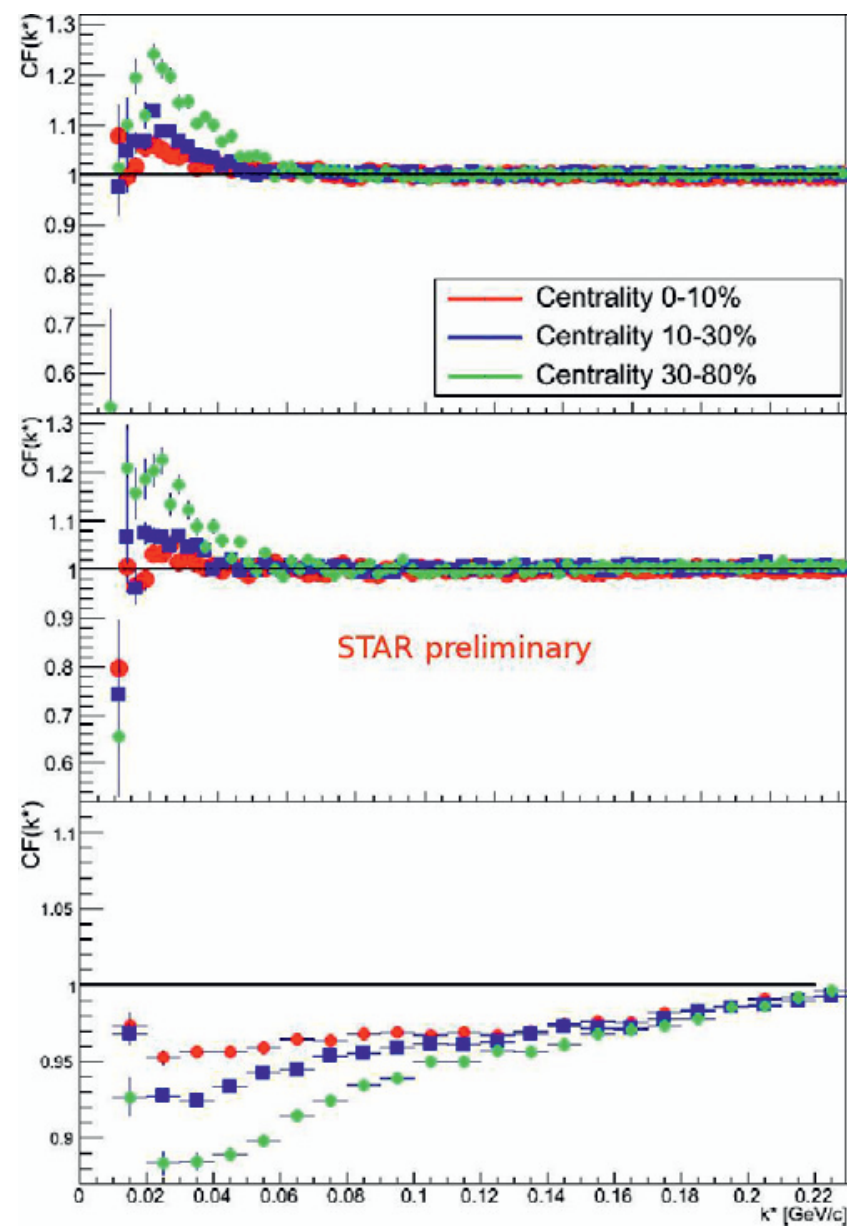

Figure 6. Corrected for the effect of residual correlations functions for proton-proton (top), antiproton-antiproton (middle), and proton-antiproton (bottom) at $\sqrt{s_{N N}}=200 \mathrm{GeV}$ for three centralities: $0-10 \%$ (red), $10-30 \%$ (blue) and $30-80 \%$ (green).

\section{Summary}

We have presented the current status of the proton-proton, antiproton-antiproton, and protonantiproton femtoscopy for $\mathrm{Au}+\mathrm{Au}$ collisions at $\sqrt{s_{N N}}=200 \mathrm{GeV}$ at STAR. Thanks to the large STAR dataset, all data could be divided into three centrality classes. The importance of the residual correlations was demonstrated for data at collision energies $\sqrt{s_{N N}}=200 \mathrm{GeV}$. The effect of residual correlations in the case of not taking them into account would be reflected in discrepancies between the source sizes estimated for identical and nonidentical combinations of protons and antiprotons.

\section{Acknowledgements}

This work was supported by the Grant of National Science Centre, Poland, No: 2012/07/D/ST2/02123. 
EPJ Web of Conferences

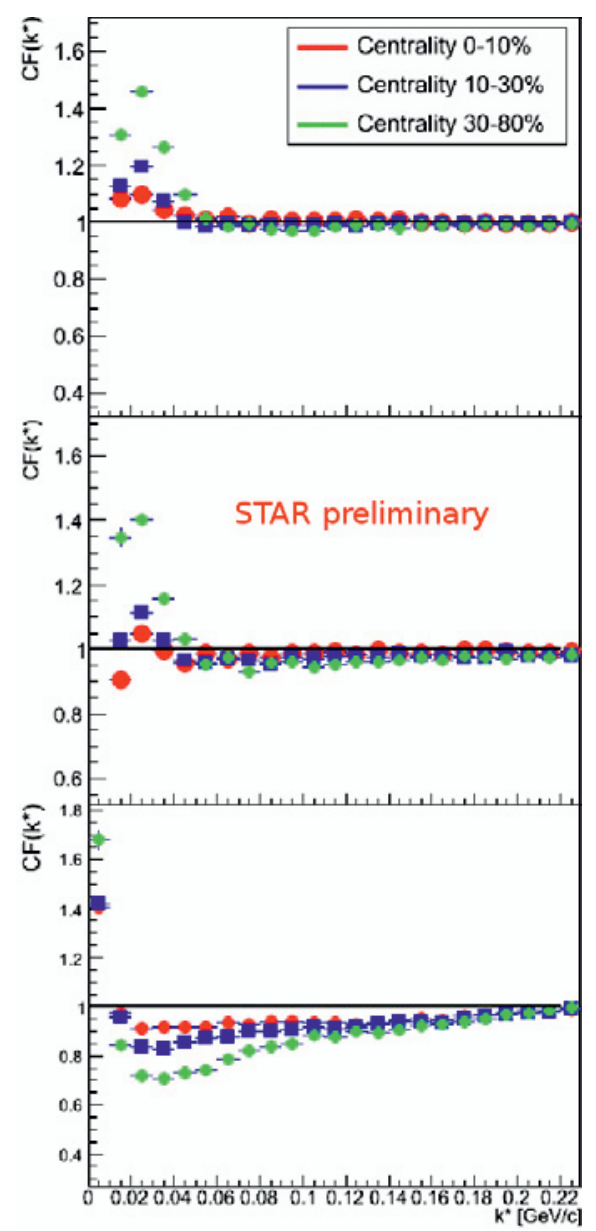

Figure 7. The measured correlation functions proton-proton (top), antiproton-antiproton (middle), and protonantiproton (bottom) at $\sqrt{s_{N N}}=200 \mathrm{GeV}$ for three centralities: $0-10 \%$ (red), $10-30 \%$ (blue) and $30-80 \%$ (green).

\section{References}

[1] G. Odyniec, J. Phys. G37, 094028 (2010); M.M.Aggarwal et al., arXiv:1007.2613v1

[2] J. Barrette et al., Phys. Rev. C60, 054905 (1999); H. Boggild et al., Phys. Lett. B458, 181 (1999); H. Appelshauser, Phys. Lett. B467, 21 (1999); R. Kotte et al., Eur. J. Phys. A23, 271 (2005)

[3] A. Kisiel, T. Taluc, W. Broniowski, W. Florkowski, Comput.Phys.Commun.174, 669 (2006)

[4] A. Kisiel, W. Broniowski, W. Florkowski, J. Pluta, Phys.Rev.C73, 064902 (2006)

[5] H.P. Gos, Eur. Phys. J. C49, 75 (2007); H.P. Zbroszczyk, Phys. Part. Nucl. Lett. vol. 8, no. 9, $931(2011)$

[6] J. Adams et al., STAR Collaboration, Phys. Rev. C74, 64906 (2006) 